\title{
O protagonismo do Enfermeiro na organização de serviços de saúde durante a pandemia da COVID-19
}

\author{
The nursing protagonism in the organization of health services during the COVID-19 pandemic \\ El protagonismo de la Enfermera en la organización de los servicios de salud durante la pandemia \\ del COVID-19
}

\section{Resumo}

Com o advento da nova pandemia pelo novo Coronavírus, razão para defrontamento mundial pelos profissionais enfermeiros na linha frente tem apresentado inquietações acerca da organização de serviços de saúde. Frente ao cenário, a necessidade de contextualizar acerca do enfrentamento das doenças respiratórias agudas crônicas, é uma das preocupações. Assim, este estudo objetiva relatar o protagonismo do enfermeiro na organização do serviço de saúde durante a pandemia da COVID-19. Com esta perspectiva, foi realizado um estudo descritivo, de abordagem qualitativa, do tipo relato de experiência, sobre o protagonismo do enfermeiro na organização do serviço de saúde durante a pandemia da COVID-19. Com o surgimento da necessidade de readaptação dos serviços para acolhimento e triagem aos casos suspeitos e implementação de Unidades para Síndromes Gripais, enfermeiros foram postos como protagonistas nos serviços de saúde no gerenciamento e prestação do cuidado em linha de frente no cenário da Pandemia. Neste sentido, os serviços de saúde passam a receber uma roupagem gerencial diferenciada para os casos de SARV-CoV-2 atendidos e observados. Condições estas que emergem para os desafios de pensar em protagonismo do enfermeiro no gerenciamento de serviços de saúde. Neste cenário da pandemia, alicerçando os saberes frente as respostas emergências para o desenvolvimento dos casos de infecção pelo COVID-19, o protagonismo do enfermeiro representa a mudança na atuação dos gestores e profissionais da saúde que lutam diariamente para a melhoria e qualidade da assistência prestada a população.

Palavras-chaves: Enfermagem; Protagonismo; Atenção primária à saúde; Pandemia; COVID-19.

\begin{abstract}
With the advent of the new pandemic for the new Coronavirus, a reason for worldwide confrontation by professional nurses on the front lines has raised concerns about the organization of health services. In view of the scenario, the need to contextualize the coping with chronic acute respiratory diseases is one of the concerns. Thus, this study aims to report the role of nurses in the organization of health services during the pandemic of COVID-19. With this perspective, a descriptive study, with a qualitative approach, of the experience report type, was carried out on the role of nurses in the organization of the health service during the pandemic of COVID-19. With the emergence of the need to re-adapt services for welcoming and tracing suspected cases and implementing Units for Flu Syndromes, nurses were placed as protagonists in health services in the management and provision of frontline care in the Pandemic scenario. In this sense, health services now receive a different managerial guise for the cases of SARV-CoV-2 attended and observed. These conditions emerge for the challenges of thinking about the role of nurses in the management of health services. In this scenario of the pandemic, basing the knowledge in the face of the emergency responses for the development of cases of infection by COVID-19, the role of the nurse represents the change in the performance of managers and health professionals who struggle daily for the improvement and quality of the assistance provided the population.
\end{abstract}

Keywords: Nursing; Protagonism; Primary health care; Pandemic; COVID-19.

\section{Resumen}

Con el advenimiento de la nueva pandemia del nuevo coronavirus, un motivo de confrontación mundial por parte de enfermeras profesionales en primera línea ha generado preocupaciones sobre la organización de los servicios de salud. Ante el escenario, la necesidad de contextualizar el afrontamiento de las enfermedades respiratorias agudas crónicas es una de las 
preocupaciones. Así, este estudio tiene como objetivo reportar el papel de las enfermeras en la organización de los servicios de salud durante la pandemia de COVID-19.Con esta perspectiva, se realizó un estudio descriptivo, con abordaje cualitativo, del tipo relato de experiencia, sobre el rol del enfermero en la organización del servicio de salud durante la pandemia de COVID19. Con el surgimiento de la necesidad de readaptar los servicios de acogida y detección de casos sospechosos y la implementación de Unidades de Síndromes Gripales, las enfermeras se colocaron como protagonistas de los servicios de salud en la gestión y prestación de atención de primera línea en el escenario de Pandemia. En este sentido, los servicios de salud reciben ahora una forma de gestión diferente para los casos de SARV-CoV-2 atendidos y observados. Estas condiciones surgen ante los desafíos de pensar el rol del enfermero en la gestión de los servicios de salud. En este escenario de la pandemia, basando el conocimiento ante las respuestas de emergencia para el desarrollo de casos de infección por COVID-19, el rol de la enfermera representa el cambio en el desempeño de los gestores y profesionales de la salud que luchan diariamente por la mejora y calidad de la atención la población.

Palabras clave: Enfermería; Protagonismo; Primeros auxilios; Pandemia; COVID-19.

\section{Introdução}

O presente estudo trata como objeto o protagonista do enfermeiro na organização de serviço de saúde. Com o advento da nova pandemia, razão para defrontamento mundial pelos profissionais enfermeiros na linha frente tem apresentado inquietações acerca da organização de serviços de saúde. Frente ao contexto, a necessidade de contextualizar acerca do enfrentamento das doenças respiratórias agudas crônicas, é uma das preocupações.

O novo coronavírus (Severe Acute Respiratory Syndromes - Related Coronavirus 2 ou SARS-CoV-2) provocou o surto identificado pela primeira vez em 31 de dezembro de 2019 em Wuhan, China (Belasco, 2020). Em 11 de março de 2020 a Organização Mundial da Saúde (OMS) declarou a disseminação da doença designada como COVID-19, configurando assim, uma pandemia (Cui, Li, \& Shi, 2019; OMS, 2020; ; Porto et al. 2021).

Cabe destacar que globalmente, até 04 de dezembro de 2020, foram confirmados 65.435.151 casos confirmados da COVID-19 e 1.510.313 mortes, enquanto isso, no Brasil foi registrado 6.487.507 casos e 175.307 óbitos. Logo, cabe ressaltar que as regiões Sudeste e Nordeste do país, apresentaram os maiores índices de casos, com 2.257.142 e 1.657 .797 casos, respectivamente (OMS, 2020b; Ministério da Saúde, 2020a).

Partindo do contexto pandêmico, o Ministério da Saúde, por meio da Portaria Ministerial no 188, de 03/02/2020, considera o estado de Emergência em Saúde Pública de importância Nacional - ESPIN (Ministério da Saúde, 2020b). Ressaltase que a Portaria Ministerial institui o Centro de Operações de Emergências em Saúde Pública como mecanismo nacional da gestão coordenada as resposta à emergência em âmbito nacional, este órgão fica sob responsabilidade da Secretaria de Vigilância em Saúde.

Logo, a Secretaria Municipal de Saúde de Maceió, a fim de organizar e otimizar as estratégias para o enfrentamento da COVID-19, em articulação com as ações de atenção à saúde dos casos suspeitos para COVID-19, implementa no cenário da Atenção Primária à Saúde (APS) Unidades de Referência para Síndromes Gripais (URSG), visando a garantia de assistência segura e responsável para a saúde da população - organizando a assistência prestada aos seus usuários, em face da alta transmissibilidade do novo vírus.

Outrossim, cabe destacar que às ações realizadas pelo profissional enfermeiro estão intrinsecamente relacionadas com o perfil gerencial para à implementação e manutenção do cuidado no enfrentamento da pandemia pela COVID-19. Assim sendo, é notória à visibilidade do enfermeiro diante à produção sistematizada no contexto de lutas - o que produz saberes e práticas experienciadas. Com esta percepção, surge à necessidade de responder ao seguinte questionamento: Qual o protagonismo do enfermeiro na organização de serviços de saúde em tempos de pandemia?

Diante o exposto, este estudo traz reflexões acerca do protagonismo do enfermeiro na organização, coordenação e manutenção do cuidado de serviços de saúde para enfrentamento da SARV-CoV-2 no cenário da APS. Logo, apresenta como objetivo, relatar o protagonismo do enfermeiro na organização do serviço de saúde durante a pandemia da COVID-19. 


\section{Metodologia}

Trata-se de um estudo de abordagem descritiva, qualitativa, do tipo relato de experiência, acerca do protagonismo do enfermeiro na organização do serviço de saúde durante a pandemia da COVID-19. Frente à nova pandemia, o enfermeiro assume o papel gestor e em URSG no enfrentamento do SARV-CoV-2 desenvolve suas habilidades e competências com efetividades para o desenrolar dos casos. Logo, cabe destacar que atividades deste cunho estão intrinsecamente relacionadas com as atribuições da categoria profissional.

Nesse sentido, as unidades vêm se destacando frente aos episódios de emergência sanitária, uma vez que utilizam atributos e princípios que regem a atenção básica, como território, o acesso, o estabelecimento de vínculos entre profissional e usuário, a integralidade do cuidado, a vigilância da população vulnerável e o monitoramento dos casos suspeitos e leves. Esses mecanismos são capazes de promover o controle do novo coronavírus e evitar complicações graves do SARS-CoV-2 (Dunlop et al., 2020; Sarti et al., 2020).

Outrossim, no cenário assistencial atual, o gerenciamento estratégico para a implementação das unidades assistenciais frente às infecções pelo SARV-CoV-2, tem sido uma forma de integração para o cuidado efetivo e qualificado. Entretanto, é notória que tal ação justifica-se pelo conjunto de conhecimentos diante o processo de trabalho conforme a teia das redes de atenção à saúde para o melhor desenvolvimento dos casos assistidos nas URSG.

Ao final deste artigo, o leitor será capaz de compreender a fundamental importância do enfermeiro na gestão pública como protagonista na organização de serviços de saúde. Entretanto, as ações desenvolvidas para o enfrentamento da COVID19, requerem integração do cuidado para as respostas emergenciais, conforme conceitualização, acerca dos desafios de pensar em protagonismo do enfermeiro no gerenciamento de serviços de saúde.

\section{Resultados e Discussão}

Diante o contexto da nova pandemia, o enfermeiro assume papéis em tempo de velocidade, mudanças e convocação para atuação meio ao enfrentamento da SARV-CoV-2. Entretanto, no cenário saúde às práticas de enfermagem buscam mudanças que ultrapassam arranjos para o desenvolvimento das ações e produção do cuidado em serviços de saúde na perspectiva de mudanças.

Tais argumentos ligados no contexto meio ao tsunami produzido na vida dos envolvidos, tem sido visto como um marco histórico que ocorreu de uma forma inesperada e tomou proporções inimagináveis - deste modo, a reestruturação dos ambientes de saúde, a readaptação dos profissionais saúde e a sobrecarga dos sistemas foram algumas das várias proporções que a nova pandemia trouxe para a sociedade.

Atualmente, o nordeste representa umas das regiões brasileiras com maior número de casos. De acordo com o painel interativo, o estado de Alagoas tem uma incidência de 2865,8/100 mil hab., o que significa 95.643 casos, em 04 de dezembro de 2020. Situações como à destacada, emergem para mudança do cenário assistencial em decorrência da gravidade da pandemia. Logo, em decorrência da gravidade da nova pandemia, estratégias para a implementação das boas práticas de cuidados, à exemplo à higiene das mãos e o isolamento social foram necessárias para conter a velocidade de propagação do vírus e atender aos que necessitam de cuidados intensivos.

Contudo, cabe destacar que o avanço mais relevante do Sistema único de Saúde (SUS) enquanto política pública se deu através da ascensão da APS nas últimas décadas frente ao principal modelo assistencial da atenção primária. Partindo da percepção, a APS se configura como fulcral na organização e na coordenação do cuidado para o enfrentamento a COVID-19 no Brasil - visto que $80 \%$ dos casos são leves e moderados e os acometidos têm a APS como primeiro acesso para cuidados (Dunlop et al., 2020; Sarti et al., 2020). 


\section{Desafios de Pensar em Protagonismo no Gerenciamento de Serviços de Saúde}

Diante deste cenário de pandemia pelo SARS-CoV-2, é notória a relevância da APS, como estratégia de enfrentamento e controle da doença, seja através da assistência longitudinal ou por meio de ações voltadas à promoção e a prevenção da saúde. Logo, desempenha um papel central, uma vez que destina seus cuidados a pessoa e a comunidade, possibilitando assim um olhar ampliado e decisões clínicas mais efetivas, voltadas ao paciente acometido ou suspeito da COVID-19 (Soeiro et al., 2020).

Entretanto, este relato traz reflexões sobre o protagonismo do enfermeiro frente à readaptação das equipes para o enfrentamento da nova pandemia, uma vez que diante do cenário brasileiro, o processo de coordenação do cuidado molda não apenas a rotina da população e das comunidades.

Assim, com o surgimento da necessidade de readaptação do serviços de saúde para o acolhimento e triagem dos casos suspeitos e implementação das Unidades para Síndromes Gripais do Município de Maceió-AL, enfermeiros são postos como protagonistas nos serviços de saúde no gerenciamento e prestação do cuidado em linha de frente no cuidado aos casos de infecção pelo COVID-19. Neste sentido, as unidades passam a receber uma roupagem gerencial diferenciada para os casos atendidos e observados, de acordo com o que se preconiza pelo Ministério da Saúde.

Vale destacar que, a assistência prestada por enfermeiros no processo de gerenciamento de unidades, em especial, às URSG é expressa por sentimentos que permeiam desde a satisfação da implantação do serviço à implementação do cuidado efetivo. Refere-se ainda, que desde a chegada à unidade, o enfermeiro está por todos os ambientes buscando artefatos para melhoria da gestão e do cuidado.

No entanto, é válido salientar que na gestão do serviço de saúde, o profissional necessita de domínio de todos os processos envolvidos - da logística a administração dos cuidados intra e extra unidade assistencial. Para tanto, no olhar aos pacientes acometidos pelo COVID-19, à integração de saberes no âmbito da classificação de risco, gerenciamento de riscos e de recursos humanos e condutas terapêuticas são os quesitos mais vislumbrados na prática.

Nessa sistemática, cabe ressaltar que os consultórios de enfermagem nas URSG fizeram-se um dos ambientes de maior valorização profissional. Com esta percepção, é importante destacar que o acolhimento frente à classificação de risco dos pacientes por meio da metodologia Fast Track para o desdobramento dos casos tornou-se um diferencial no contexto da atenção à saúde.

Tal metodologia engloba aspectos que identificam as necessidades do paciente na chegada do serviço e logo passam a ser avaliados pelo profissional enfermeiro com um bundle "Ficha atendimento da unidade de triagem para Síndromes Gripais". Neste ínterim, estudos como o de Lima et al. (2021) defendem que o uso de protocolos como aliança para a melhoria da assistência e a tomada de decisão baseada em suporte teórico, conceitual, científico e padronizado estão interligados para a qualidade e segurança do paciente - garantindo assim, um desempenho profissional diferenciado.

Esta integração metodológica proporciona ao profissional possibilidades diante à oferta do cuidado. No modelo de APS, os profissionais enfermeiros, são considerados agentes de mudança no processo de trabalho em saúde - estabelecendo condição singular entre instrumentos e sujeitos-objetos das intervenções, bem como, realizando a criação e manutenção do vínculo, seguindo as diretrizes de ações em redes, frente aos princípios da equidade e integralidade do cuidado.

Vale destacar que, os profissionais correm um risco elevado de contrair a infecção, principalmente enquanto direciona cuidados à pacientes confirmados para o COVID-19. Logo, deve-se atentar para o dimensionamento de pessoal enquanto enfermeiro gestor e traçar estratégias logísticas de realocação para estas situações. Além disso, o papel do enfermeiro é um destaque. Com esta percepção, estudos como o de Gallotti et al.(2021) reiteram que no processo assistencial o respaldo em ciência e foco no cuidado integral e efetivo estão associados a melhoria e eficácia do trabalho. 
Para a operacionalização, aconteceram treinamentos na unidade com uma equipe de enfermeiros atuantes nas estratégias de implementação do serviço e de educação permanente, com abordagens variáveis - fluxos de funcionamento, biossegurança, protocolos e rede de apoio do serviço - em pequenos grupos e por categoria profissional e em outros momentos, com a equipe de plantão do dia para orientações, reorganização e manutenção dos fluxos. Este foi um método factível ao aprendizado de profissionais de saúde. Labegalini et al.(2021) reforçam que tais aspectos estão relacionados a importância da prevenção, da organização e do planejamento das ações.

Com este olhar alicerçado na pratica assistencial, à liderança de equipe deve reconhecer desde o princípio que o gerenciamento de risco do serviço envolve elevados níveis de pressão psicológicas. Logo, deve-se pontuar neste movimento que às negociações das prioridades e organizações das estratégias são aspectos inerentes que conseguem manter equilibrada às ações coordenadas entre os níveis hierárquicos.

Face ao exposto, identifica-se que no momento, este tipo de relato de experiência bem-sucedidas de profissionais da enfermagem são sistematicamente pouco expressos ao protagonismo do enfermeiro, uma vez que, meio à linha de frente se permite à excelência do trabalho no cenário saúde em tempos de pandemia.

\section{Conclusão}

Neste cenário da pandemia, alicerçando os saberes frente as respostas emergências para o desenvolvimento dos casos de infecção pelo COVID-19, o protagonismo do enfermeiro representa a mudança na atuação dos gestores e profissionais da saúde que lutam diariamente para a melhoria e qualidade da assistência prestada a população. Destaca-se ainda, que em interfaces ao cuidado, o protagonismo do enfermeiro é motivo de orgulho a existência da enfermagem.

Logo, o trabalho desempenhado de forma singular, ainda mais em meio à pandemia que vivemos, garante aspectos de manutenção e gerenciamento, bem como, minimização dos riscos existentes a partir da reorganização dos serviços de saúde para padronização dos atendimentos que cuide em tempo hábil e escalas ininterruptas. Desse modo, razões como estas, são pilares para a exclusividade que motivam a equipe para uma atenção equânime e integral do ser em tempos de pandemia.

Desta forma, este estudo demonstra a importância do protagonismo do enfermeiro no gerenciamento de serviços de saúde frente aos desafios e avanços no cenário de pandemia pelo COVID-19. Nesta concepção, espera-se que novos estudos acerca da problemática sejam desenvolvidos para melhor compreensão do fenômeno.

\section{Referências}

Cui, J., Li, F., \& Shi, Z-Li (2019). Origin and evolution of pathogenic coronaviruses. Nature Reviews Microbiology. 17,181-192. https://pubmed.ncbi.nlm.nih.gov/30531947/

Ministério da Saúde (2020a). Painel Coronavírus. Brasília, DF, 2020a. https://covid.saude.gov.br/

Ministério da Saúde (2020b). Portaria no 188, de 3 de Fevereiro de 2020. Brasília, DF, 2020b. https://www.in.gov.br/web/dou/-/portaria-n-188-de-3-defevereiro-de-2020-241408388

Dunlop, C. et al. (2020). The coronavirus outbreak: the central role of primary care in emergency preparedness and response. BJGP Open. https://bjgpopen.org/content/bjgpoa/4/1/bjgpopen20X101041.full.pdf

Fehr, A., \& Perlman, S. (2015). Coronaviruses: an overview of their replication and pathogenesis. Methods Mol Biol, 1282, 1-23. https://pubmed.ncbi.nlm.nih.gov/25720466/

Gallotti, F. C. M. et al. (2021). Formação do enfermeiro na perspectiva do cuidado integral e trabalho em equipe. Research, Society and Development, 10(1). https://rsdjournal.org/index.php/rsd/article/view/11724/10457

Labegalini, C. M. G. et al. (2021). O processo de enfrentamento da pandemia de COVID-19 na perspectiva de profissionais da Enfermagem. Research, Society and Development, 10(1). https://rsdjournal.org/index.php/rsd/article/view/11252/10245

Lima, R. M. L. S. et al. (2021). Conhecimento dos enfermeiros acerca da importância do uso de protocolos de cuidados: Discurso do sujeito coletivo. Research, Society and Development, 10(1). https://rsdjournal.org/index.php/rsd/article/view/11186/10356 
Research, Society and Development, v. 10, n. 1, e48110112014, 2021

(CC BY 4.0) | ISSN 2525-3409 | DOI: http://dx.doi.org/10.33448/rsd-v10i1.12014

OMS (2020a). Organização Munidal De Saúde. Linha do tempo da resposta da OMS ao COVID-19. Genebra: OMS. https://coronavirus.saude.gov.br/linhado-tempo

OMS (2020b). Organização Munidal De Saúde. Painel da Doença de Coronavírus da OMS (COVID-19). Genebra: OMS. https://coronavirus.saude.gov.br/

Pereira, A. S., et al. (2018). Metodologia da pesquisa científica. UAB/NTE/UFSM.: em: https://repositorio.ufsm.br/bitstream/handle/1/15824/Lic_Comp utacao_Metodologia-Pesquisa-Cientifica.pdf?sequence=1

Porto, E. F., et al. (2021). Mortalidade por Covid-19 no Brasil: perfil sociodemográfico das primeiras semanas. Research, Society and Development, 10(1). https://rsdjournal.org/index.php/rsd/article/view/11588/10593

Sarti, T. D. et al. (2020). Qual o papel da Atenção Primária à Saúde diante da pandemia provocada pela COVID-19? Epidemiol. Serv. Saude, Brasília, 29(2), 2020166. http://scielo.iec.gov.br/scielo.php?script=sci_arttext\&pid=S1679-49742020000200043\&lng=pt\&nrm=iso\&tlng=en

Soeiro, R. E. et al.(2020). Atenção Primária à Saúde e a pandemia de COVID-19: reflexão para a prática. InterAm J Med Heath. 3 , e202003 